\title{
Amino acid disorders
}

\author{
Ermal Aliu $^{1}$, Shibani Kanungo ${ }^{2}$, Georgianne L. Arnold ${ }^{1}$ \\ ${ }^{1}$ Children's Hospital of Pittsburgh, University of Pittsburgh School of Medicine, Pittsburgh, PA, USA; ${ }^{2}$ Western Michigan University Homer Stryker \\ MD School of Medicine, Kalamazoo, MI, USA \\ Contributions: (I) Conception and design: S Kanungo, GL Arnold; (II) Administrative support: S Kanungo; (III) Provision of study materials or \\ patients: None; (IV) Collection and assembly of data: E Aliu, GL Arnold; (V) Data analysis and interpretation: None; (VI) Manuscript writing: All \\ authors; (VII) Final approval of manuscript: All authors. \\ Correspondence to: Georgianne L. Arnold, MD. UPMC Children's Hospital of Pittsburgh, 4401 Penn Avenue, Suite 1200, Pittsburgh, PA 15224, USA. \\ Email: georgianne.arnold4@chp.edu.
}

\begin{abstract}
Amino acids serve as key building blocks and as an energy source for cell repair, survival, regeneration and growth. Each amino acid has an amino group, a carboxylic acid, and a unique carbon structure. Human utilize 21 different amino acids; most of these can be synthesized endogenously, but 9 are "essential" in that they must be ingested in the diet. In addition to their role as building blocks of protein, amino acids are key energy source (ketogenic, glucogenic or both), are building blocks of Kreb's (aka TCA) cycle intermediates and other metabolites, and recycled as needed. A metabolic defect in the metabolism of tyrosine (homogentisic acid oxidase deficiency) historically defined Archibald Garrod as key architect in linking biochemistry, genetics and medicine and creation of the term 'Inborn Error of Metabolism' (IEM). The key concept of a single gene defect leading to a single enzyme dysfunction, leading to "intoxication" with a precursor in the metabolic pathway was vital to linking genetics and metabolic disorders and developing screening and treatment approaches as described in other chapters in this issue. Amino acid disorders also led to the evolution of the field of metabolic nutrition and offending amino acid restricted formula and foods. This review will discuss the more common disorders caused by inborn errors in amino acid metabolism.
\end{abstract}

Keywords: Phenylketonuria (PKU); amino acids; intoxication; metabolic formula; dietary protein

Submitted Dec 07, 2018. Accepted for publication Dec 07, 2018.

doi: $10.21037 / \mathrm{atm} .2018 .12 .12$

View this article at: http://dx.doi.org/10.21037/atm.2018.12.12

\section{Introduction}

Amino acids are the building blocks of proteins, including structural proteins and enzymes. They also form the backbones of critical nitrogen-based compounds such as cytochromes, heme, hormones, melanin, neurotransmitters, nucleotides/nucleic acids and others. Amino acids are used efficiently by the body by multiple mechanisms such as recycling, transamination, or energy production. In healthy individuals consuming a regular diet, only a small amount of protein intake is wasted in urine or feces. Proteins not only serve as structural or working compounds, they can also be metabolized to create energy. When needed, the carbon skeletons can be used to produce glucose or its derivatives (such as glycogen or fatty acids) to support plasma glucose levels or provide energy during fasting.

Dietary proteins are key sources of essential amino acids in mammals and humans, while non-essential amino acids can by synthesized endogenously. Humans utilize 21 different amino acids with the $21^{\text {st }}$ amino acidselenocysteine, being unique that it does not follow the canonical pathways of the other 20 proteinogenic amino acids synthesis, but has an important role in various cellular processes such as oxidative stress, signaling pathways, thyroid hormone metabolism, protein folding, and selenium homeostasis (1).

Nine amino acids (histidine, isoleucine, leucine, lysine, methionine, phenylalanine, threonine, tryptophan, valine) 
are considered as "essential", i.e., essential in the diet as humans are unable to synthesize them endogenously.

Most of the non-essential amino acids such as alanine, arginine, asparagine, aspartate, glutamate, glutamine, glycine, proline and serine, are synthesized from glucose; while tyrosine is synthesized from the metabolism of phenylalanine, and cysteine from the metabolism of methionine (2).

Alkaptonuria, an inborn error of amino acid metabolism, has the distinction of being the first metabolic disorder described. Sir Archibald Garrod investigated why the urine of some individuals turned black, working out the defect in tyrosine metabolism at homogentisic acid oxidase. Thus was born a new field of medicine "Inborn Errors of Metabolism" (IEM) and the one-gene-one enzyme concept $(3,4)$. Subsequently, the identification of a defect in phenylalanine metabolism, phenylketonuria (PKU) led to evolution of Newborn Screening (NBS) and its success as discussed in another topic 'Newborn screening and the changing face of inborn errors of metabolism in the United States' of this issue. Through developments in the study of PKU, the concept of dietary restriction of the offending amino acid as a treatment strategy led to subsequent evolution of the field of "Metabolic Nutrition" (5). This review will further discuss common inborn errors of amino acid metabolism, their clinical presentation, diagnostic evaluation, treatment and management approaches.

\section{Disorders of phenylalanine and tyrosine}

Phenylalanine is an essential amino acid and tyrosine is a semi-essential amino acid. In humans, tyrosine is synthesized from phenylalanine through phenylalanine hydroxylase, which adds a hydroxyl group to the aromatic ring. Tyrosine is catabolized via several steps forming acetoacetate (ketogenic) and fumarate (glucogenic) as end-products. The hyperphenyalaninemias and the hypertyrosinemias are reviewed in detail in the Online Metabolic and Molecular Bases of Inherited Disease (OMMBID) $(6,7)$. The biochemical pathway for phenylalanine and tyrosine metabolism is illustrated in Figure 1.

PKU (OMIM \#261600) is one of the earliest defined inborn errors of metabolism, and has a well-described history. It has been called "the epitome of human biochemical genetics". A number of historical summaries are published on this disorder $(8,9)$. PKU was first identified in 1934 by Asbjørn Følling when he found elevated phenylketones in the urine of two siblings with intellectual disability and unusual odor. The mechanism of the toxicity of phenylalanine is not fully understood. Treatment by a phenylalanine-restricted diet was first described by Horst Bickel in 1953 and early and continuous treatment appears to prevent the intellectual disability. The majority of cases are caused by deficiency of the enzyme phenylalanine bydroxylase, with a few percent of case from deficiency of synthesis or recycling of the biopterin cofactor. PKU is inherited in an autosomal recessive fashion. If untreated during infancy and childhood, PKU causes intellectual disability, seizures, "mousy" odor, fair hair/skin, eczema, and other findings. The incidence varies depending on the population, with the highest incidence in the Turkish population (approximately 1:2,600), and as high as 1:10,000 in Northern European and East Asian populations) (10).

Classical PKU was originally defined as phenylalanine levels above $1,200 \mu \mathrm{mol} / \mathrm{L}(20 \mathrm{mg} / \mathrm{dL})$, however this distinction is now somewhat outdated as phenylalanine levels much lower are still associated with sub-optimal cognitive and neurological outcomes. Current treatment guidelines recommend maintaining phenylalanine levels between 120-360 $\mu \mathrm{mol} / \mathrm{L}(2-6 \mathrm{mg} / \mathrm{dL})$ throughout life (9). The current mainstay of treatment is dietary restriction of phenylalanine with supplementation of tyrosine. However, the diet is highly restrictive, and few can remain compliant in adolescence and adulthood, leading to executive functioning deficits that impair education, employment and interpersonal relationships. Newer treatments are now beneficial for a subset of patients. Patients with residual enzyme activity may benefit from supplementation with the biopterin cofactor sapropterin (currently approved for ages four and older), which can reduce phenylalanine levels in some. Pegvalaise is an injected form of phenylalanine ammonium lyase recently approved for adults with PKU that can, in many cases, normalize blood phenylalanine levels. Experimental treatments include gene therapy or hepatocyte transplantation.

PKU also has the distinction of being the original newborn screening disorder. Recognizing that early and universal diagnosis could prevent the intellectual disability led Robert Guthrie to develop the bacterial inhibition assay in 1963 that enabled widespread newborn screening. It should be remembered that phenylalanine levels are lower in the first days of life as the infant has not yet been introduced to protein feeds, thus mild elevations of phenylalanine on the newborn screen are frequently higher on follow-up testing. Newborn screening programs typically use the ratio of phenylalanine to specific amino acids to 


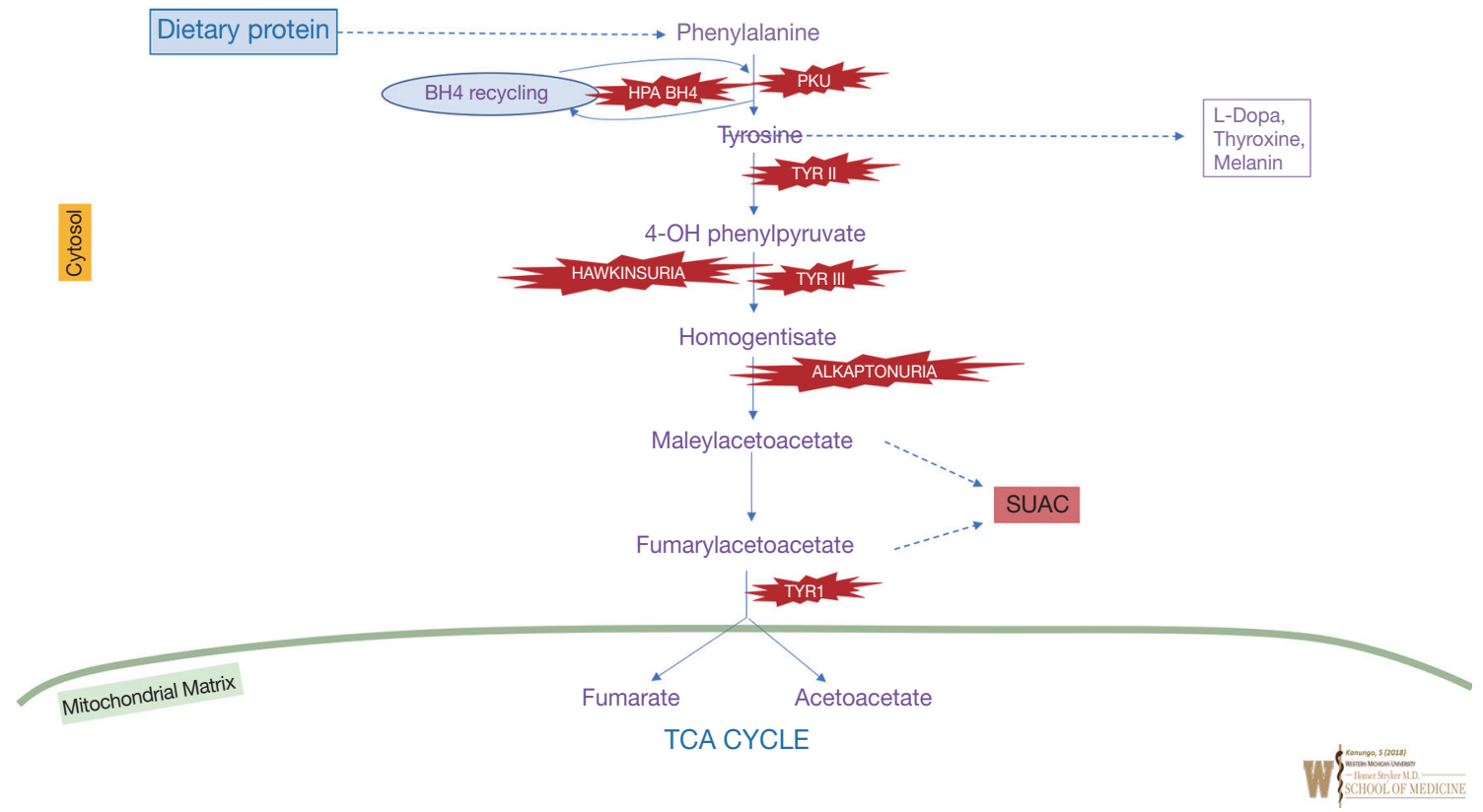

Figure 1 Overview of phenylalanine and tyrosine metabolism. Enzyme deficiencies are shown in the red star shapes. Green line represents mitochondrial membrane. SUAC, succinylacetone; BH4, tetrahydrobiopterin; HPA, hyperphenylalaninemia, PKU, phenylketonuria; TYR, tyrosinemia.

identify hyperphenylalaninemia as early as 24 hours of life, for example a phenylalanine to tyrosine ratio $>3$ is helpful diagnostically. Newborn screening positive infants should also undergo screening for defects in biopterin synthesis or recycling as these can also lead to elevated phenylalanine levels.

\section{Maternal PKU}

During pregnancy, females with PKU should maintain phenylalanine levels $<360 \mu \mathrm{mol} / \mathrm{L}(6 \mathrm{mg} / \mathrm{dL})$ since higher levels are associated with teratogenic effects such as microcephaly, congenital heart defects, IUGR, intellectual disability and other poor outcomes (11).

\section{Biopterin defects}

A small proportion of hyperphenylalaninemia is due to defects in the synthesis or recycling of the biopterin cofactor for the phenylalanine hydroxylase enzyme. Because this cofactor is shared with tyrosine hydroxylase and tryptophan hydroxylase, affected patients typically also have impaired production of dopamine and serotonin, and exhibit neurological abnormalities even with phenylalanine restriction (12).
Tyrosinemia type II (OMIM \#276600) (aka RichnerHanhart syndrome) is a disorder of the first step of tyrosine degradation in the enzyme tyrosine aminotransferase. Tyrosine and its metabolites (4-hydroxyphenylpyruvate, 4-hydroxyphenyllactate, 4-hydroxyphenylacetate) are increased in blood, CSF and urine (7). The disorder is inherited in an autosomal recessive manner.

Typically, without treatment the levels of tyrosine in plasma are $>1,200 \mu \mathrm{mol} / \mathrm{L}$. In untreated cases, patients present in the first one to two years of life with eye findings and most commonly go on to develop skin and neurological findings. Tyrosine crystal deposition in the corneal epithelial cells leads to photophobia, excessive lacrimation and burning sensation. Skin abnormalities develop mainly in pressure area like palms and soles, beginning as blisters or erosions progressing to nonpruritic, hyperkeratotic papules and plaques. Neurological outcome varies from normal development to intellectual disability (mild to severe), in some cases with seizures, tremor and ataxia. The reason for neurological complications remains unclear. This disorder is rare, with an incidence of less than $1 / 250,000$.

Definitive diagnosis can be established by gene analysis. Dietary restriction of tyrosine and phenylalanine is recommended to maintain tyrosine levels in plasma in 
the $200-500 \mu \mathrm{mol} / \mathrm{L}$ range while maintaining adequate phenylalanine levels. In pregnancy tighter control might be beneficial to further lower the fetal risks associated with hypertyrosinemia.

The disorder is detectable on newborn screening. Elevation of tyrosine on the newborn screen due to tyrosinemia type II must be differentiated from tyrosinemia type I or type III (see below), liver disease (which commonly also presents with elevations of methionine and other evidence of liver dysfunction), and transient tyrosinemia of the newborn, which is usually self-limited but can occasionally linger for a number of months after birth (13). Transient tyrosinemia of the newborn is a common biochemical finding in neonates, particularly with prematurity. The cause is suspected to be immaturity of 4-hydroxyphenylpyruvate dioxygenase, possibly in the presence of high protein intake and/or relative ascorbate deficiency. Although usually regarded as benign, there are reports of association with neonatal lethargy and some mild intellectual disability. When plasma tyrosine levels remain elevated and other causes of elevated tyrosine are ruled out, treatment for transient tyrosinemia of the newborn including protein restriction to $2 \mathrm{~g} / \mathrm{kg} /$ day and ascorbic acid $50-200 \mathrm{mg} /$ day supplementation for 1-2 weeks can be helpful.

Tyrosinemia type III (OMIM \#276710) is a rare autosomal recessive disorder caused by pathogenic variants in the enzyme 4-hydroxyphenylpyruvate dioxygenase (HPD gene). Tyrosine and its metabolites (4-hydroxyphenylpyruvate, 4-hydroxyphenyllactate, 4-hydroxyphenylacetate) are elevated in blood and urine. The most common observed complication is intellectual disability, which can vary from mild to severe. Other findings include psychomotor delays, intermittent ataxia, or drowsiness. It is very rare, with unknown prevalence. The cause for neurological complications is unknown but possibly related due to elevated tyrosine. Genetic testing can help confirm the diagnosis. Treatment involves tyrosine-restriction to maintain tyrosine plasma levels in the $200-500 \mu \mathrm{mol} / \mathrm{L}$ range. It is a rare cause of elevated tyrosine on the newborn screen (14).

Hawkinsinuria (OMIM \#140350) is also due to deficiency of the 4-hydroxyphenylpyruvate dioxygenase enzyme, but in this case is attributable to dominantly inherited milder changes in the HPD gene. Hawkinsin (4-dihydroxycyclohexyl acetic acid) is formed from tyrosine metabolite precursors, with only minimal to no elevation of tyrosine. In the first years of life affected patients can present with failure to thrive and metabolic acidosis, but this disorder appears to be asymptomatic later in life. It is rare with unknown prevalence. Only a few cases have been described. The presence of hawkinsin in urine amino acid analysis is diagnostic, and can be confirmed by genetic testing. When the diagnosis is made in infancy, restoration of breast feeding has been associated with clinical improvement, and in some cases dietary restriction of tyrosine and supplementation with vitamin $\mathrm{C}$ have been helpful (7).

Alkaptonuria (OMIM \#203500) is a recessive disorder caused by pathogenic changes in the HGD gene, encoding homogentisate dioxygenase. It is notable historically as the first inborn error of metabolism described by Garrod. Patients accumulate homogentisic acid, and by the third decade of life experience pigmentation of connective tissue (particularly visible in the sclerae or ear cartilage), arthritis of joints and spine, cardiac valve involvement, kidney and prostate stones. In children it can be diagnosed due to darkened urine when exposed to air. The arthritis mimics osteoarthritis involving large weight bearing joints and spine. Joint replacement surgeries and cardiac valve involvement is typically seen in half of the patients by $6^{\text {th }}$ decade. In United States the prevalence is estimated to be 1:250,000-1,000,000 live births, more common in the Dominican Republic and in northwestern Slovakia. Elevated homogentisate by quantification in the urine or bi-allelic pathogenic variants in $H G D$ can confirm the diagnosis. In the past several therapies (dietary restriction of tyrosine and phenylalanine, high dose Vitamin C or oral bisphosphonate) have been tried but have not been proven to slow/reverse the progression of disorder. Recently nitisinone was proposed as a potential therapeutic agent; it results in biochemical improvement but demonstrates mixed results in already symptomatic patients $(15,16)$.

Tyrosinemia type I (OMIM \#276700) is a life-threatening disorder of tyrosine metabolism caused by pathogenic variants in FAH gene, which encodes fumarylacetoacetase (the last step in tyrosine degradation pathway). The enzyme defect causes the accumulation of precursors maleylacetoacetate and fumarylacetoacetate, and their byproducts (succinylacetone and succinylacetoacetate) are toxic to cells, particularly liver and kidney. Succinylacetone is also a potent inhibitor of 5-Aminolevulinic acid dehydratase leading to accumulation of 5-ALA, which is neurotoxic and thought to be the cause of the acute (porphyria-like) neurological crises. It is inherited in an autosomal recessive manner. The most severe form presents early, often in the first six months of life with liver disease progressing to liver failure if untreated. Later presentations include liver dysfunction and renal tubular acidosis, growth 
failure, hypophosphatemic rickets and porphyria-like symptoms. For those who survive past infancy, the natural course if left untreated is a progressive disease ultimately leading to cirrhosis and/or hepatocellular carcinoma.

Succinylacetone (SUAC) in blood or in urine is pathognomonic, but is not universally detected on urine organic acid analysis, thus should be specifically requested if this diagnosis is considered. Plasma tyrosine elevations can be minimal; thus, plasma amino acids alone may fail to provide diagnosis. Incidence is about 1:100,000-120,000 births. In the Saguenay-Lac Saint-Jean region of Quebec the prevalence is $1: 1,846$ live births due to founder effect (c. $1062+5 \mathrm{G}>\mathrm{A}$ is seen in $87 \%$ of pathogenic variants). Newborn screening by measurement of succinylacetone is available in many, but not all newborn screening programs. Because plasma tyrosine level elevations can be minimal, this disorder can be missed on newborn screening unless the program also detects succinylacetone.

The treatment of choice is 2-(2-nitro-4-trifluoromethylbenzyol) 1,3 cyclohexanedione (NTBC) also known as nitisinone and should be started immediately after the diagnosis is confirmed. It inhibits a more proximal step in the tyrosine degradation pathway (4-hydroxyphenylpyruvic acid dioxygenase enzyme), thus preventing the formation of the toxic metabolites. Since nitisinone will result in elevation of plasma tyrosine levels, dietary restriction of tyrosine and phenylalanine are required to maintain levels in the desirable range (serum tyrosine levels of 200-400 $\mu \mathrm{mol} / \mathrm{L}$ while maintaining adequate phenylalanine levels. Treatment with nitisinone decreases the risk for hepatocellular cancer, but patients require ongoing monitoring. Patients diagnosed before nitisinone was developed required orthotopic liver transplantation (OLT) $(7,17,18)$.

\section{Disorders of branched chain amino acids}

The branched chain amino acids (leucine, isoleucine, valine) are essential amino acids in humans, accounting for $20 \%$ of the amino acids in muscle proteins and play an important role in protein synthesis and turnover.

Maple Syrup Urine Disease (MSUD) (OMIM \#248600) is a disorder of branched chained amino acid (BCAA) catabolism (see Figure 2). It is an autosomal recessive inborn error of metabolism caused by pathological variants in the subunits of the enzyme complex involved in the second common step in branched chain keto-acid metabolism [BCKDHA (OMIM \#608348), BCKDHB (OMIM \#248611), DBT (OMIM \#248610), or DLD (OMIM \#238331)] (see Figure 2). Five phenotypes have been describedclassic, intermediate, intermittent, thiamine responsive, and dihydrolipoyl dehydrogenase (E3)-deficient. In classical MSUD newborns show signs of the disease within the first days to months of life. Elevated leucine causes cerebral edema and intoxication, initially with nonspecific symptoms such as poor feeding/irritability, then progressing to lethargy, intermittent apnea, cerebral edema, coma and death. Later or milder forms can present with encephalopathy during episodic metabolic decompensation, particularly during intermittent illnesses. The prevalence is $1: 185,000$ in the general population but in certain Mennonite communities it is reported to be 1:380 due to a founder effect $(19,20)$.

Plasma amino acid will reveal significant elevations of valine, leucine and isoleucine, as well as l-alloisoleucine (pathognomonic marker for MSUD). The diagnosis can be confirmed by molecular testing. The "maple syrup" odor (from accumulation of 2-hydroxyisoleucine) is not reliably obvious in many cases (and often best appreciated in cerumen).

Treatment of acute crisis may require hemodialysis to rapidly lower plasma leucine levels. Other important elements of treatment include reversing catabolism (short term protein restriction, providing at least 1.25 of daily energy requirement, with $40-50 \%$ as fat), followed by supplementation of valine and isoleucine and gradual addition of leucine to fulfill minimal requirements for this essential amino acid. Due to cerebral edema risk, hypotonic fluids should be avoided. Dietary restriction of leucine (goal is to maintain serum levels between $150-300 \mu \mathrm{mol} / \mathrm{L}$ ) with valine and isoleucine supplementation is the mainstream of chronic therapy (21).

OLT removes dietary restrictions and ongoing concern for acute metabolic crises and is becoming more popular with MSUD patients (22). Since about half of BCAA metabolism occurs in skeletal muscle, explanted livers of patients with MSUD have been used in "domino transplants" as liver donors.

MSUD is detectable on newborn screening, but the infant may become ill before the screening results are completed. False positive elevations of branched chain amino acids can be attributable to hyperalimentation, catabolism, or other factors, but a newborn screen suspicious for MSUD should be considered a metabolic emergency until the differential diagnosis is ruled out.

Hypervalinemia (OMIM 277100) and byperleucineisoleucinemia (OMIM 238340) are biochemical findings of 


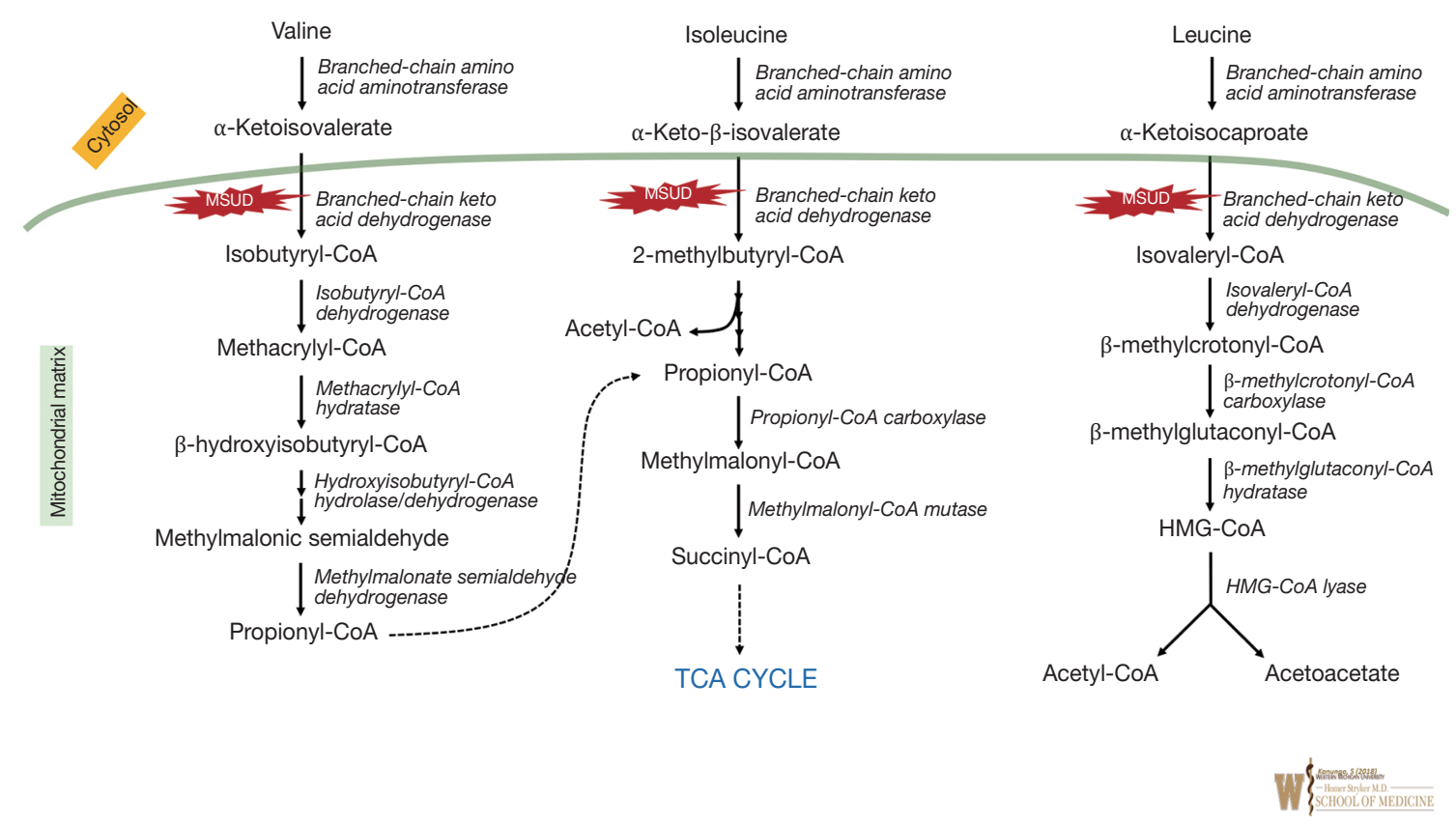

Figure 2 Overview of Branched-chain amino acid catabolism. Enzyme names are represented in italics and corresponding intramitochondrial enzyme deficiencies are shown in the red star shapes. Green line represents mitochondrial membrane. MSUD, maple syrup urine disease.

uncertain significance caused by BCT1 (OMIM 113520) and BCT2 (OMIM 113530). One patient has been reported who had both hypervalinemia and hyperleucineisoleucinemia with brain white matter abnormalities, who had improvement on vitamin B6 supplementation.

\section{Disorders of the sulfated amino acids (methionine and cysteine)}

Methionine is an essential amino acid and provides the most common starting codon for protein translation. The differential diagnosis of elevated plasma methionine includes tyrosinemia type I (due to liver disease), citrin deficiency (see urea cycle disorders) homocystinuria, and the disorders of homocysteine synthesis. Methionine is converted to homocysteine via the intermediates s-adenosylmethionine (SAM) and s-adenosylhomocysteine (SAH). Homocysteine levels are tightly regulated, either by returning homocysteine to methionine via methionine synthase using the methylene tetrahydrofolate reductase (MTHFR) pathway (the remethylation pathway), or further metabolized to cystathionine by the enzyme cystathioninebeta-synthase, then subsequently to cysteine, sulfite, and finally, sulfate. Figure 3 illustrates various IEMs identified at multiple enzymatic steps in this pathway (23).

Isolated hypermethioninemia is due to defects in the pathway of the conversion of methionine to to homocysteine. This conversion requires three steps: conversion of methionine to s-adenosylmethionine [by methionine adenosyl transferase (MAT)], conversion of s-adenosylmethionine to s-adenosylhomocysteine by glycine methyltransferase, and conversion of $\mathrm{S}$-adenosylhomocysteine to homocysteine by $\mathrm{S}$-adenosylhomocysteine hydrolase (SAHH). Inborn errors are reported in all three of these steps in the pathway.

MAT I/III deficiency (OMIM 250850) is associated with defects in the conversion of methionine to s-adenosylmethionine due to pathogenic variants in the MATA1A gene (OMIM \#250850). It is the most common of the three disorders in this pathway. Both dominant and recessive forms are described. In most patients it appears to be a benign biochemical phenotype. However, in the recessive form when plasma methionine levels exceed $800 \mu \mathrm{mol} / \mathrm{L}$ it can be associated with neurological deficits/ demyelination. Diagnosis is made by ascertainment of hypermethioninemia (on plasma amino acid analysis) in the absence of hyperhomocysteinemia (requires definitive testing as homocysteine is unstable on standard plasma 


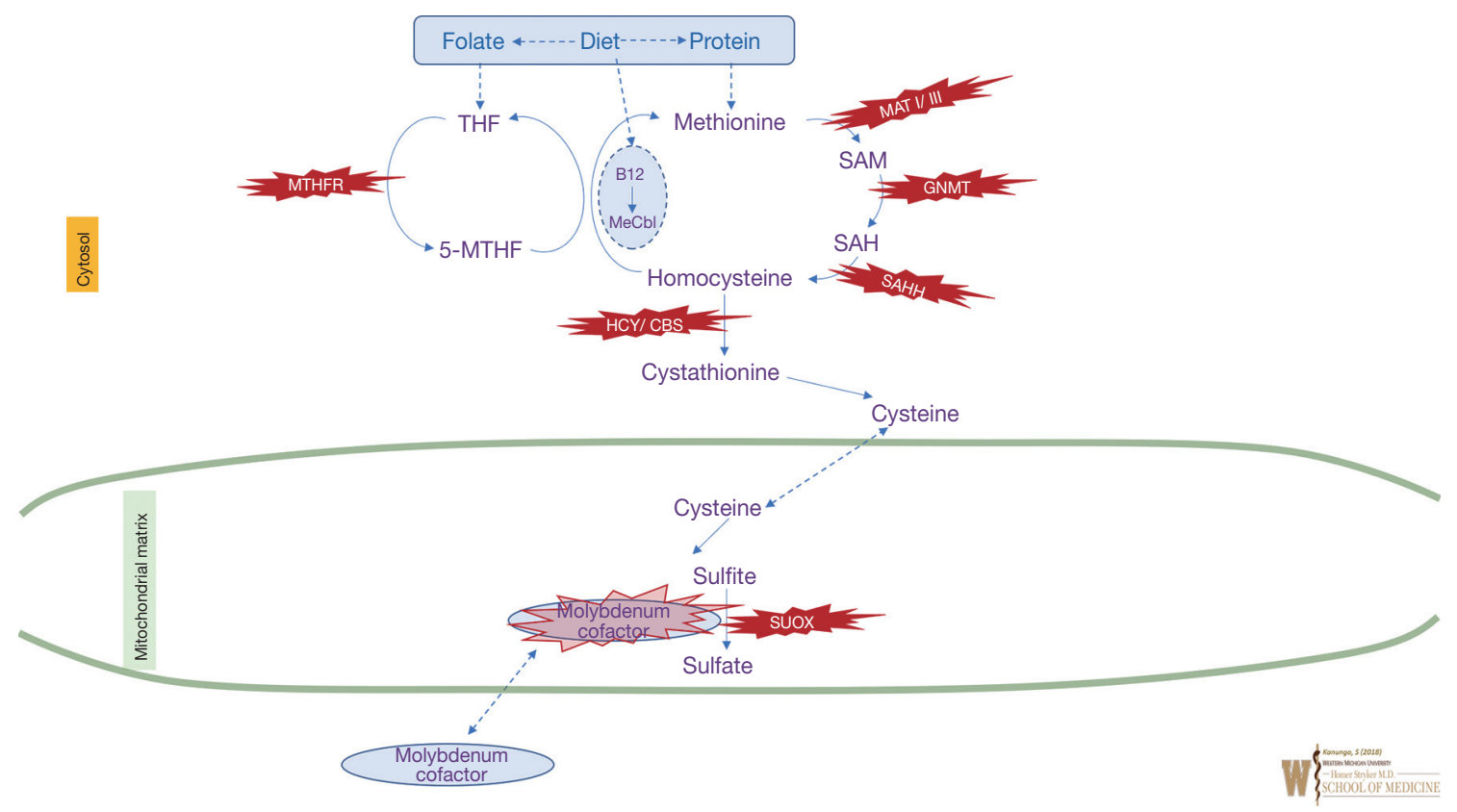

Figure 3 Overview of sulfated amino acid metabolism. Enzyme names are represented in italics and corresponding intra-mitochondrial enzyme deficiencies are shown in the red star shapes. Green line represents mitochondrial membrane. B12, vitamin B12; CBS, cystathionine beta synthase; HCY, Homocystinuria; MeCbl, methylcobalamin; MAT, methionine S-adenosyltransferase; SAM, S-adenosylmethionine; SAH, S-adenosylhomocysteine; SAHH, S-adenosylhomocysteine hydrolase; MTHFR, methylene tetrahydrofolate reductase; THF, tetrahydrofolate; SUOX, sulfite oxidase.

amino acid analysis). The instability of homocysteine on plasma amino acid analysis requires a separate, definitive assay for plasma homocysteine. Treatment is not required unless plasma methionine level is very high, in which case methionine restriction may be needed. This disorder is detected incidentally on newborn screening, as the screen measures methionine (not homocysteine) levels, due to the instability of plasma homocysteine. In the presence of neurological abnormalities or liver disease, it may be necessary to consider the other two disorders in this pathway (below).

Glycine N-Methyltransferase deficiency (OMIM \#606664) is associated with a defect in the conversion of s-adenosylmethionine to s-adenosylhomocysteine. It is a very rare autosomal recessive disorder caused by GNMT deficiency, with only few cases reported. Findings include persistent mildly elevated serum liver transaminases and mild hepatomegaly. Biochemical findings include elevated methionine and S -adenosylmethionine (SAM) (24).

S-adenosylhomocysteine bydrolase deficiency (OMIM 613752) has been reported twice with elevated plasma methionine and s-adenosylmethionine without significant homocystinuria (HCY). Clinical features included liver disease, neurologic abnormalities, white matter abnormalities, and elevated CK. One case had molecular testing of the ACHY gene, and had bi-allelic pathogenic variants. He showed some improvement with dietary restriction of methionine, and supplementation with phosphatidyl choline and creatine (25).

Homocystinuria (classical homocystinuria, OMIM \#236200) is an autosomal recessive disorder caused by pathological variants in CBS gene which encodes cystathionine beta-synthase. Classical homocystinuria (as well as the more severe remethylation disorders associated with hyperhomocysteinemia) is characterized by involvement of the eye (ectopia lentis associated with abnormal collagen crosslinking in the zonules, progressive myopia), skeletal system (Marfanoid habitus-tall, slender with increased upper/lower ratio of the extremities, scoliosis, pectus, stiff joints), vascular system (thromboembolism), and CNS (developmental delays/intellectual disability). Patients have increased risk for coronary heart disease. Pregnancy increases the post-partum risk for thromboembolism events. Prevalence of classical homocystinuria is estimated to be 
$1: 200,000$ to $1: 335,000$ in the United States, but in certain areas of the world is much higher (e.g., Qatar 1:1,800, Norway 1:6,400).

Plasma levels of methionine are elevated on amino acid analysis. Plasma levels of homocysteine are elevated but may not be appreciated on plasma amino acid analysis unless the blood is immediately deproteinized, thus specific analysis of plasma homocysteine is indicated when this disorder is suspected. The diagnosis is confirmed biochemically or by the presence of bi-allelic pathogenic variants in the cystathionine beta synthase (CBS) gene.

Patients with classical homocystinuria with specific mutations may be responsive to supplementation with the cofactor pyridoxine (vitamin B6). At diagnosis patients should undergo a pyridoxine challenge. Standard treatment includes dietary restriction of methionine and supplementation with cysteine to maintain plasma homocysteine levels below $50 \mu \mathrm{mol} / \mathrm{L}$. Betaine increases the remethylation of homocysteine back to methionine and may be helpful. Maternal homocystinuria is not teratogenic, but increase the mother's elevated risk of thromboembolism, and low molecular weight heparin should be considered to be given in the last trimester and 6 weeks post-partum.

Newborn screening for homocystinuria is accomplished by measuring methionine levels in the newborn as plasma homocysteine is not stable for this analysis. Elevation of methionine on newborn screening should be followed by specific ascertainment of plasma homocysteine, as well as B12 and folate levels $(26,27)$.

Homocystinuria can also be caused by failure to remethylate homocysteine back to methionine. As noted, previously, homocysteine levels are tightly regulated. In addition to conversion of homocysteine to beta cystathionine, homocysteine is also actively remethylated back to methionine. This process involves vitamin B12, methionine synthase, and MTHFR.

Defects in vitamin $B 12$ metabolism involve the modification of cobalamin to form methylcobalamin (MeCbl). This process includes multiple steps, with inborn errors in various steps referred to by their complementation group. Defects in complementation groups $\mathrm{C}, \mathrm{D}, \mathrm{F}, \mathrm{J}$ and $\mathrm{X}$ also result in decreased formation of adenosyl B12 and some forms of methylmalonic acidemia. Nutritional deficiency of vitamin B12 can also result in mild elevations of homocysteine, but not to the degree seen in pathologic metabolic defects (28).

Defects in MTHFR (OMIM \#236250): the enzyme MTHFR is involved in folate metabolism and the remethylation of homocysteine to methionine. Severe genetic defects result in signs and symptoms characteristic of classic homocystinuria. Neurologic abnormalities are common including seizures, microcephaly, severe intellectual disability, and psychiatric disorders.

The common polymorphisms in MTHFR (C677T and A1298C) result in some decrease in enzyme expression, but are not inborn errors of metabolism. They can present with mild hyperhomocystinemia, but not to the degree that causes homocystinuria.

In the case of remethylation defects, plasma methionine is low to low-normal, while plasma homocysteine levels are significantly elevated. Patients with remethylation defects may respond to large doses of cobalamin $(1 \mathrm{mg} /$ day or more of hydroxocobalamin injections), and to betaine. Because homocystinuria due to a remethylation defect is not associated with hypermethioninemia, it is NOT detectable by routine newborn screening unless accompanied by other cobalamin complementation defects, i.e., methylmalonic acidemia).

The detoxifiction of sulfite to the non-toxic sulfate is the final step in the catabolism of the sulfated amino acids. The enzyme responsible is sulfite oxidase. Currently, two inborn errors of sulfite oxidase activity are described, isolated deficiency of the sulfite oxidase enzyme itself, and deficiency if its cofactor, the molybdenum cofactor (29).

Molybdenum cofactor deficiency (OMIM \#252150 \& $252160)$ is an autosomal recessive disorder affecting the biosynthesis of the molybdenum cofactor, which is required for the enzymes - sulfite oxidase, aldehyde oxidase and xanthine dehydrogenase. The loss of activity of sulfite oxidase cause by the cofactor deficiency appears to be the most clinically significant of the three catalyzed enzymes. The classic presentation is intractable neonatal seizures with abnormal EEG (often a burst suppression pattern, often accompanied by structural CNS anomalies, with later intellectual disability and (after one year) ectopia lentis. Later onset or milder presentations are also reported. The disorder is rare, but is also likely under-diagnosed.

Although the classic metabolite s-sulfocysteine can be detected on some amino acid analyses, it is often not discerned given its position on the chromatogram and lack of awareness of the disorder. Thus, specific testing is indicated for s-sulfocysteine. Dipstick of fresh urine for sulfite can also be helpful in diagnosis. Serum uric acid is also typically low (secondary to xanthine dehydrogenase deficiency). Genetic testing can confirm the diagnosis. Treatment with intravenous pyranopterin monophosphate leads to considerably improved outcome in some, but not 
all patients with Type A deficiency (30).

Isolated sulfite oxidase deficiency (OMIM \#272300) is a recessive disorder caused by defects in the gene SUOX. It is strikingly similar to molybdenum cofactor deficiency in presentation, with neonatal seizures, intellectual disability and later ectopia lentis, but in this case serum uric acid is normal. Treatment with methionine and cysteine supplementation may result in minimal improvement, but no substantial treatments are available (31). Neither disorder is detectable on newborn screening at this time.

\section{Disorders of glycine metabolism}

Glycine encephalopathy (aka non-ketotic hyperglycinemia) (OMIM \#605899) is a disorder of the glycine cleavage system. Glycine accumulates in cerebrospinal fluid and other body tissues. Most affected patients present in the newborn period with intractable seizures, commonly with a burst-suppression pattern on EEG, and survivors have profound intellectual disability. A neonatal variant form presents with progressive lethargy and apnea. About 15\% present later in infancy to adulthood. Occasionally, the developmental outcome is milder. The disorder is inherited in an autosomal recessive manner. It is an important cause of neonatal seizures. Incidence is as high as 1:55,000 in Northern European populations, but it is likely the disorder is under-diagnosed since it may not be appreciated without ascertaining glycine levels (on amino acid analysis) in cerebrospinal fluid.

Diagnosis is suspected based on an elevated ratio of $\mathrm{CSF}$ /plasma glycine. Plasma glycine is not always elevated. Confirmation can include genetic testing for the (at least) 3 proteins comprising the glycine cleavage enzyme complex. Enzyme assay is possible on liver tissue. There is currently no highly effective treatment. Sodium benzoate (which complexes with glycine to form hippuric acid) can enhance glycine excretion, and dextromethorphan can have an anticonvulsant effect, presumably by attenuating the overexcitation of the NMDA receptor in the CNS. However, in severe neonatal cases, the efficacy of these treatments is minimal.

Newborn screening is being proposed for this disorder based on elevation of plasma glycine levels. However the sensitivity is unclear as plasma glycine is not uniformly elevated in this disorder $(32,33)$.

Other rare amino acid disorders include histidinemia, Hartnup disease, hyperlysinemia, formiminotransferase deficiency, 2 aminoadipic semialdehyde dehydrogenase (antiquin deficiency), prolidase deficiency, proline oxidase, deficiency, 5-oxoprolinase deficiency etc. will not be reviewed in this article and can be found in www.omim.org.

\section{Acknowledgements}

None.

\section{Footnote}

Conflicts of Interest: The authors have no conflicts of interest to declare.

\section{References}

1. Schmidt RL, Simonović M. Synthesis and decoding of selenocysteine and human health. Croat Med J 2012;53:535-50.

2. Lieberman M, Peet A. Marks' basic medical biochemistry : a clinical approach. Available online: https://www.lww. co.uk/marks-basic-medical-biochemistry-content-contentcontent

3. Garrod A. The Incidence of Alkaptonuria : A Study in Chemical Individuality. Lancet 1902;160:1616-20.

4. Beadle GW, Tatum EL. Genetic Control of Biochemical Reactions in Neurospora. Proc Natl Acad Sci U S A 1941;27:499-506.

5. Bickel H, Gerrard J, Hickmans EM. Influence of phenylalanine intake on phenylketonuria. Lancet (London, England) 1953;265:812-3.

6. Donlon J, Sarkissian C, Levy H et al. Hyperphenylalaninemia: Phenylalanine Hydroxylase Deficiency. In: Beaudet AL, Vogelstein B, Gibson KM, et al. editors. The Online Metabolic and Molecular Bases of Inherited Disease. New York, NY: The McGraw-Hill Companies, Inc., 2014.

7. Mitchell GA, Grompe M, Lambert M, et al. Hypertyrosinemia. In: Beaudet AL, Vogelstein B, Kinzler KW, et al. editors. The Online Metabolic and Molecular Bases of Inherited Disease. New York, NY: The McGrawHill Companies, Inc., 2014.

8. Camp KM, Parisi MA, Acosta PB, et al. Phenylketonuria Scientific Review Conference: State of the science and future research needs. Mol Genet Metab 2014;112:87-122.

9. Vockley J, Andersson HC, Antshel KM, et al. Phenylalanine hydroxylase deficiency: diagnosis and management guideline. Genet Med 2014;16:188-200.

10. Regier DS, Greene CL. Phenylalanine Hydroxylase 
Deficiency. GeneReviews®. University of Washington, Seattle, 1993.

11. Waisbren SE, Rohr F, Anastasoaie V, et al. Maternal Phenylketonuria: Long-term Outcomes in Offspring and Post-pregnancy Maternal Characteristics. JIMD Rep 2015;21:23-33.

12. Blau N, Thöny B, Hyland K, et al. Disorders of Tetrahydrobiopterin and Related Biogenic Amines. In: Beaudet AL, Vogelstein B, Gibson KM, et al. editors. The Online Metabolic and Molecular Bases of Inherited Disease. New York, NY: The McGraw-Hill Companies, Inc., 2014.

13. Levy HL, Shih VE, Madigan PM, et al. Transient Tyrosinemia in Full-Term Infants. JAMA 1969;209:249.

14. Ellaway CJ, Holme E, Standing S, et al. Outcome of tyrosinaemia type III. J Inherit Metab Dis 2001;24:824-32.

15. Introne WJ, Gahl WA. Alkaptonuria. In: Adam MP, Ardinger HH, Pagon RA, et al. editors.

SourceGeneReviews ${ }^{\circledR}$ [Internet]. Seattle (WA): University of Washington, Seattle, 1993-2018.

16. Ranganath LR, Khedr M, Milan AM, et al. Nitisinone arrests ochronosis and decreases rate of progression of Alkaptonuria: Evaluation of the effect of nitisinone in the United Kingdom National Alkaptonuria Centre. Mol Genet Metab 2018;125:127-34.

17. Sniderman King L, Trahms C, Scott CR. Tyrosinemia Type I. GeneReviews ${ }^{\circledR}$ [Internet]. Seattle (WA): University of Washington, Seattle, 1993-2018.

18. Chinsky JM, Singh R, Ficicioglu C, et al. Diagnosis and treatment of tyrosinemia type I: a US and Canadian consensus group review and recommendations. Genet Med 2017;19(12).

19. Chuang DT, Shih VE, Max Wynn R. Maple Syrup Urine Disease (Branched-Chain Ketoaciduria). In: Beaudet AL, Vogelstein B, Gibson KM, et al. editors. The Online Metabolic and Molecular Bases of Inherited Disease. New York, NY: The McGraw-Hill Companies, Inc., 2014.

20. Strauss KA, Puffenberger EG, Morton DH. Maple Syrup Urine Disease [Internet]. GeneReviews ${ }^{\circledR}$. University of Washington, Seattle, 1993.

21. Frazier DM, Allgeier C, Homer C, et al. Nutrition management guideline for maple syrup urine disease: An evidence- and consensus-based approach. Mol Genet Metab 2014;112:210-7.

22. Mazariegos G V, Morton DH, Sindhi R, et al. Liver transplantation for classical maple syrup urine disease: long-term follow-up in 37 patients and comparative United Network for Organ Sharing experience. J Pediatr 2012;160:116-21.e1.

23. Harvey Mudd S, Levy HL, Kraus JP. Disorders of Transsulfuration. In: Beaudet AL, Vogelstein B, Kinzler $\mathrm{KW}$, et al. editors. The Online Metabolic and Molecular Bases of Inherited Disease [Internet]. New York, NY: The McGraw-Hill Companies, Inc., 2014.

24. Barić I, Erdol S, Saglam H, et al. Glycine N-Methyltransferase Deficiency: A Member of Dysmethylating Liver Disorders? JIMD Rep 2017;31:101-6.

25. Baric I, Fumic K, Glenn B, et al. S-adenosylhomocysteine hydrolase deficiency in a human: a genetic disorder of methionine metabolism. Proc Natl Acad Sci U S A 2004;101:4234-9.

26. Sacharow SJ, Picker JD, Levy HL. Homocystinuria Caused by Cystathionine Beta-Synthase Deficiency [Internet]. GeneReviews ${ }^{\circledR}$. University of Washington, Seattle, 1993.

27. Morris AAM, Kožich V, Chakrapani AB, et al. Guidelines for the diagnosis and management of cystathionine betasynthase deficiency. J Inherit Metab Dis 2017;40:49-74.

28. Sloan JL, Carrillo N, Venditti CP et al. Disorders of Intracellular Cobalamin Metabolism [Internet]. GeneReviews@. University of Washington, Seattle, 1993.

29. Johnson JL, Duran M. Molybdenum Cofactor Deficiency and Isolated Sulfite Oxidase Deficiency. In: Beaudet AL, Vogelstein B, Gibson KM, et al. editors. The Online Metabolic and Molecular Bases of Inherited Disease. New York, NY: The McGraw-Hill Companies, Inc., 2014.

30. Atwal PS, Scaglia F. Molybdenum cofactor deficiency. Mol Genet Metab 2016;117:1-4.

31. Bindu PS, Nagappa M, Taly AB et al. Isolated Sulfite Oxidase Deficiency [Internet]. GeneReviews ${ }^{\circledR}$. University of Washington, Seattle, 1993.

32. Hamosh A, Johnston M V. Nonketotic Hyperglycinemia. In: Beaudet AL, Vogelstein B, Gibson KM, et al. editors. The Online Metabolic and Molecular Bases of Inherited Disease [Internet]. New York, NY: The McGraw-Hill Companies, Inc., 2014.

33. Van Hove J, Coughlin C, Scharer G. Glycine Encephalopathy [Internet]. GeneReviews®. University of Washington, Seattle, 1993.

Cite this article as: Aliu E, Kanungo S, Arnold GL. Amino acid disorders. Ann Transl Med 2018;6(24):471. doi: 10.21037/ atm.2018.12.12 